(C) 1996 IEEE. Personal use of this material is permitted. However, permission to reprint/republish this material for advertising or promotional purposes or for creating new collective works for resale or redistribution to servers

or lists, or to reuse any copyrighted component of this work in other works must be obtained from the IEEE.

\title{
Beamline Smoothing of the Advanced Photon Source*
}

\author{
H. Friedsam, M. Penicka, S. Zhao \\ Argonne National Laboratory \\ 9700 South Cass Avenue \\ USA - Argonne, Illinois 60439
}

\section{Abstract}

This paper outlines a general beamline smoothing concept based on the use of First Principle Component analysis. Beamline smoothing is commonly used for the detection of blunders in the positioning of beam elements and to provide a smooth particle beam path with the fewest adjustments to individual beam components. It also provides the data for assessment of the achieved positioning quality.

\section{INTRODUCTION}

During the last three years the Advanced Photon Source (APS) has been under construction at Argonne National Laboratory. The APS is a 7-GeV synchrotron light source which is being used for basic research in material science, chemistry, physics, biology, and medicine.

The APS consists of a 70m-long linear accelerator, a positron accumulator ring (PAR), a synchrotron ring (SY) with a circumference of $368 \mathrm{~m}$, and the storage ring (SR) with a circumference of $1104 \mathrm{~m}$ (Fig. 1). The 40m electron linac uses $200-\mathrm{MeV}$ electrons for the production of positrons. In the $30 \mathrm{~m}$-long linear accelerator section following the electron linac, the particles gain a mass of $450 \mathrm{MeV}$ before entering the positron accumulator ring. From there the beam is injected into the booster synchrotron which accelerates the positrons from $450 \mathrm{MeV}$ to $7 \mathrm{GeV}$ before entering the storage ring. The storage ring can accommodate up to $68 \mathrm{x}$-ray beamlines.

\section{ALignMENT TOLERANCES}

The global alignment tolerance requires the positioning of all beam components within an envelop of $\pm 5 \mathrm{~mm}$ of their ideal position. The circumference of the storage ring should be within $\pm 20 \mathrm{~cm}$ of the nominal design value.

The most demanding relative alignment tolerance is required for the positioning of the storage ring multipoles. The maximum tolerable transverse and vertical displacements for these beam components must be within $\pm 0.15 \mathrm{~mm}[1]$.

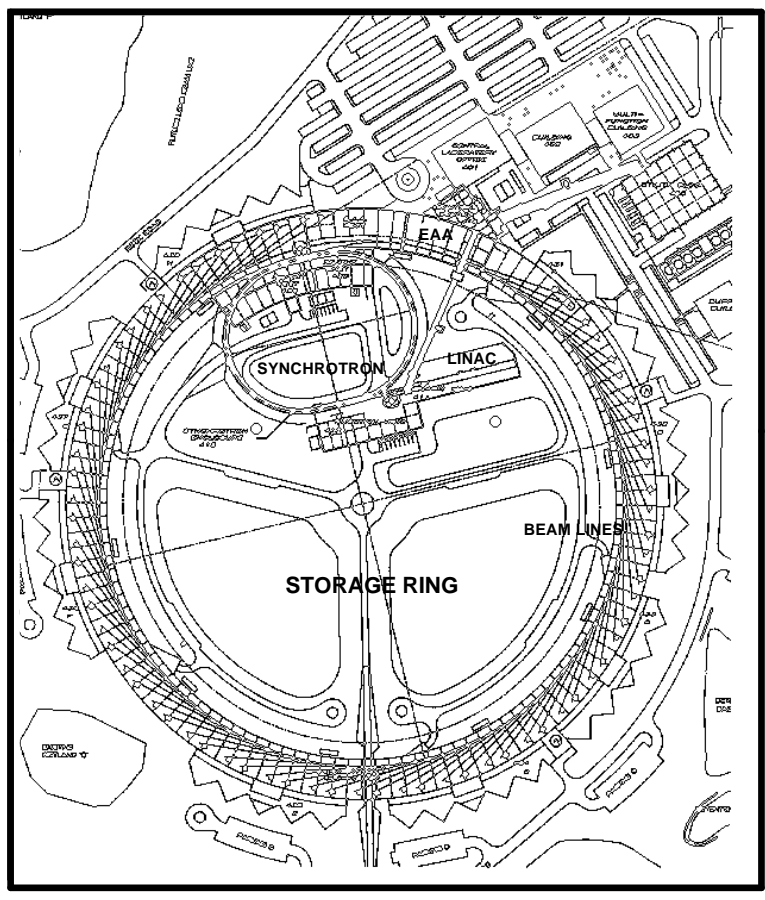

Fig. 1 APS site overview

\section{THE BEAMLINE SMOOTHING CONCEPT}

The global positioning accuracy for the beam components is determined by systematic and random errors. When performing least squares adjustments of the survey measurements, the data is assumed to be free of systematic errors; otherwise the concept of least squares is not applicable. Many precautions are taken to reduce the effects of systematic errors during the measurement process by applying special survey procedures [2].

Using error propagation for the measurements between two primary control points [3] yields the absolute positioning envelope as shown in Fig. 2.

The absolute error envelope is minimal in the vicinity of the connection to the primary control network points and reaches its maximum halfway between these points. The actual smooth beamline represented by the magnet fiducial marks meanders within the boundaries of the absolute error envelope as depicted by the dashed line in Fig. 2. The ideal reference line given by the lattice coordinates for each beam component is shown as a solid straight line connecting the two primary control points.

\footnotetext{
* Work supported by US DOE Office of Basic Energy Sciences under Contract No. W-31-109-ENG-38.
} 


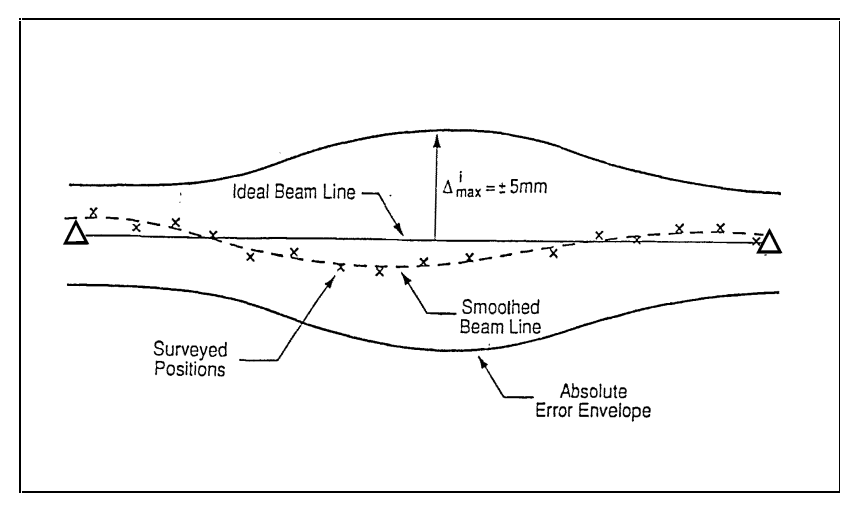

Fig. 2 Absolute error envelope

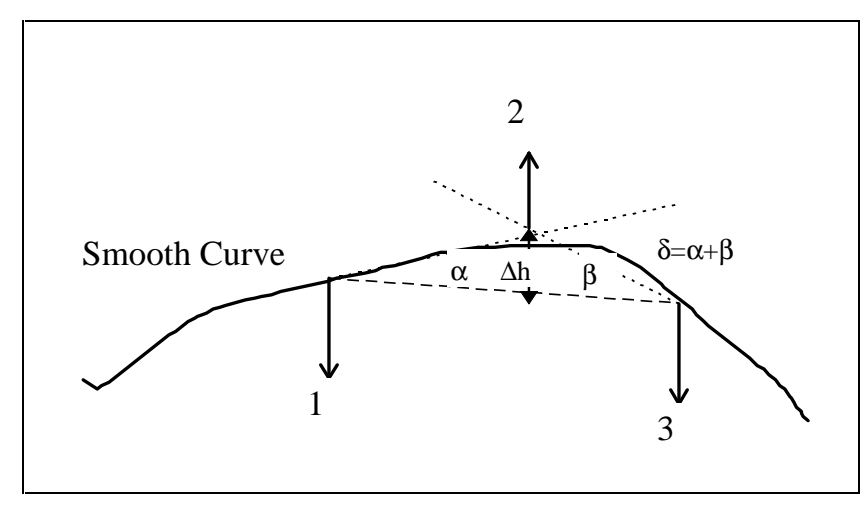

Fig. 3 Smoothness criteria

\section{AlignMENT RESUltS}

The task of the alignment team is to ensure a smooth particle beamline. It is of no importance to the operation of the accelerator wether this curve is identical to the ideal reference line or one of many smooth curves within the boundaries of the absolute error envelope as long as smoothness between adjacent beam components is guaranteed. Therefore the goal of the relative alignment step is to obtain a smooth beamline with a minimum number of required adjustments to individual beam elements.

Different concepts have been proposed to tackle this task. See for instance the approach taken by DESY to perform the relative alignment of HERA [4] or the route taken by CERN for smoothing the SPS beamline [5].

The approach used for smoothing the APS booster synchrotron and storage ring beamlines is based on a development originating at SLAC for the relative alignment of the SLC [6]. It is a general beamline smoothing concept based on First Principle Component analysis and is applicable to almost all bemlines independent of the beamline geometry. The beamline geometry is of no importance because the algorithm operates on the differences between the ideal and actual measured position of the beam component.

Principal curves are defined as smooth, one-dimensional curves passing through the middle of a p-dimensional data set, minimizing the sum of the perpendicular distances from the data points to the curve. The result is a non-parametric curve with a shape suggested by the data. The curves are found in an iterative process starting with the principal component line [7].

A smoothness criteria has to be defined that will stop the iteration process or the curve would approach each data point, thus defeating the purpose of smoothing. In the case of the APS, all multipoles are supposed to be aligned to $\pm 0.15 \mathrm{~mm}$ relative to each other. That means that the middle component of three successive beam elements numbered 1, 2 and 3 in Fig. 3, should not deviate from a straight line connecting the end components by more than $\Delta \mathrm{h}$, which is $\pm 0.15 \mathrm{~mm}$ for the APS.
After the completion of the storage ring girder alignment the positions of all quadrupoles, sextupoles and dipoles were measured once more using laser trackers [8]. In order to obtain the connection between individual tracker setups, additional temporary control points were installed and measured. The data was analyzed using a bundle adjustment routine developed at SLAC. The differences between the resulting magnet positions from the bundle adjustment and the ideal locations were then subjected to the smooth curve fitting operation.

The results of the smoothing process can be seen in Figs. 4 and 5 for the transverse and vertical deviations from the ideal position. One can see that the smooth curve deviates from the ideal position by $\pm 4 \mathrm{~mm}$, within the confines of the envelop for the absolute positioning of beam components.

Finally Figs. 6 and 7 show the histograms for the deviations of the multipoles from the smooth beamline. All components have been placed within the $2 \sigma$ range. Out of 680 multipoles, only $3 \%$ in the transverse direction, and $2 \%$ in the vertical direction have been placed in the $1 \sigma$ to $2 \sigma$ range. The achieved relative position tolerances are $\pm 0.09 \mathrm{~mm}$ transverse and $\pm 0.07 \mathrm{~mm}$ vertical, well within the specified tolerance level.

\section{SUMMARY}

Considering that a relative positioning accuracy of $\pm 0.09 \mathrm{~mm}$ in the transverse direction and $\pm 0.07 \mathrm{~mm}$ in the vertical direction was achieved, and taking into account the standard deviation for the determination of the magnetic center versus the mechanical center of $\pm 0.075 \mathrm{~mm}$, the position between magnetic centers is estimated to be within $\pm 0.125 \mathrm{~mm}[9]$.

The successful alignment of the APS storage ring is also documented by the rapid progress in the commissioning program. Shortly after the start of the storage ring commissioning phase, the beam was transported around the whole ring using only four horizontal and three vertical correctors. Multiple turns have been recorded in the mean time and rf capture of the beam has been demonstrated. 


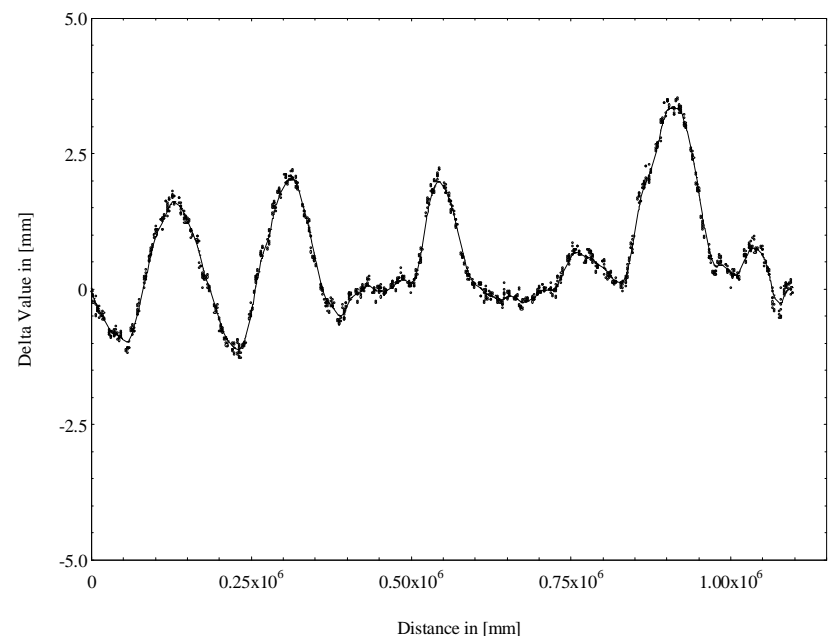

Fig. 4 Transverse displacements

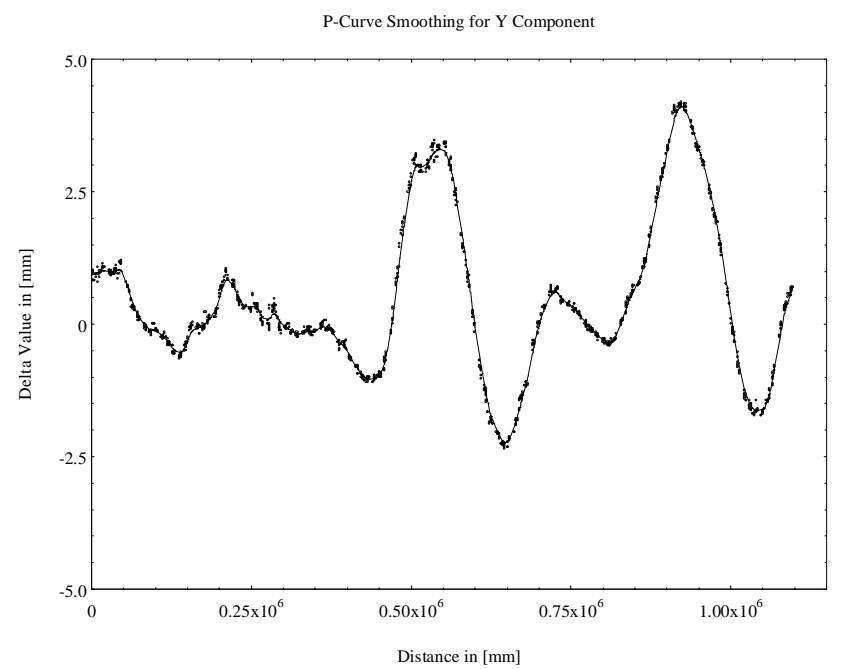

Fig. 5 Vertical displacements

\section{REFERENCES}

[1] 7-GeV Advanced Photon Source Conceptual Design Report, Argonne National Laboratory, ANL-87-15, (1987).

[2] R. Ruland, Accelerator and Transport Line Survey and Alignment, (Stanford Linear Accelerator Center, California, SLAC Pub.-5672, 1991).

[3] H. Friedsam, J. Penicka, S. Zhao, Survey and Alignment Report on the Primary Control Network for the APS, Light Source Note LS-220, (1993).

[4] W. Schwarz, Die Justierung von Teilchenbeschleunigern, (Allgemeine Vermessungs Nachrichten, Heft 1, 1990).

[5] M. Mayoud, "Applied Metrology for LEP, " in CERN Accelerator School Applied Geodesy for Particle Accelerators, (CERN, Switzerland, April 1987).

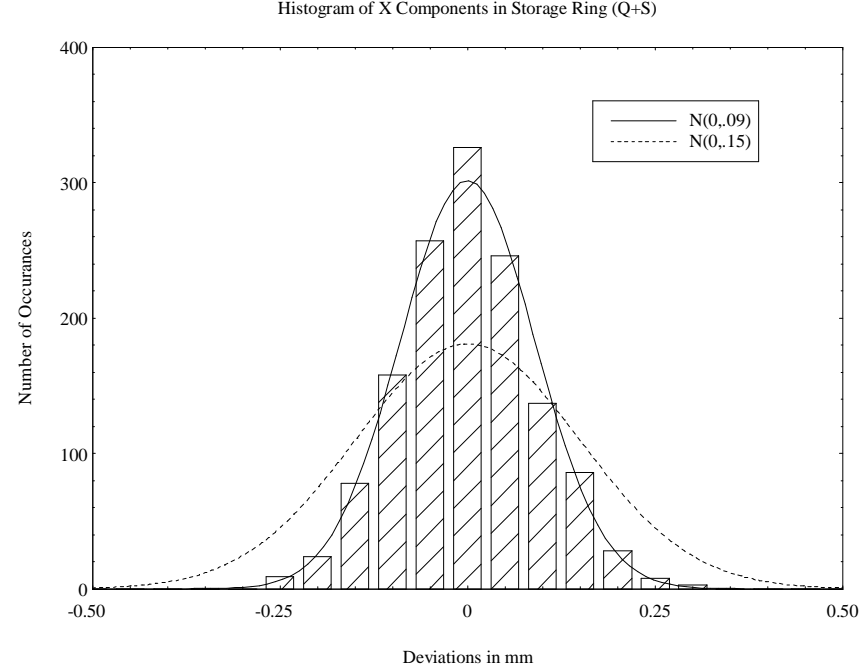

Fig. 6 Histogram of the transverse displacements of all storage ring multipoles

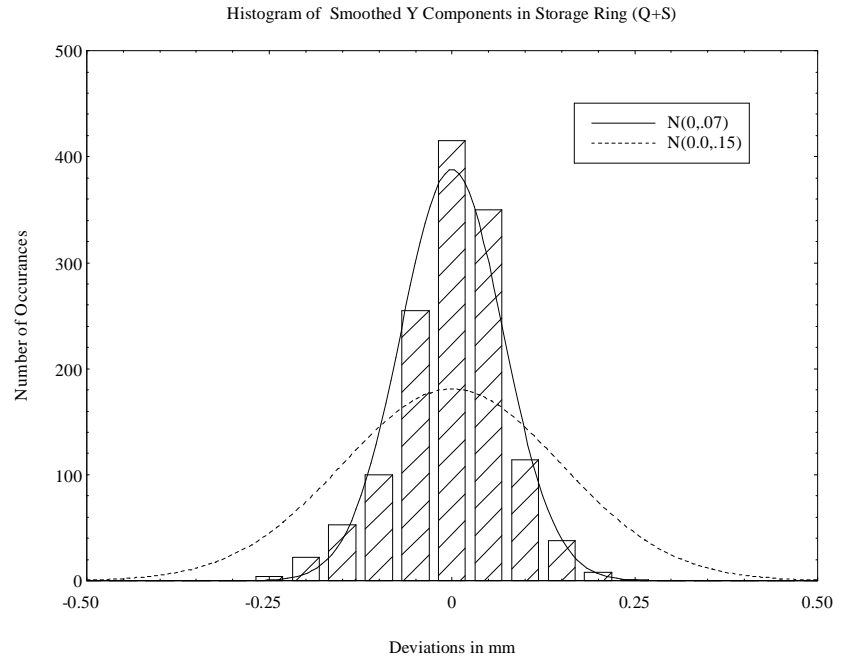

Fig. 7 Histogram of the vertical displacements of all storage ring multipoles

[6] H. Friedsam, W. Oren, "The Application of the First Principal Curve Analysis Technique to Smooth Beamlines, "in Proceedings of the First International Workshop on Accelerator Alignment, (Stanford Linear Accelerator Center, California, 1989)

[7] Trevor Hastie, Principal Curves and Surfaces, (Stanford Linear Accelerator Center, California, SLAC Pub.-276, 1984).

[8] H. Friedsam, "A New Accelerator Alignment Concept using Laser Trackers," in Proceedings of the Fourth European Particle Accelerator Conference, (London, England, 1994).

[9] S. Kim, D. Carnegie, C. Doose, R. Hogrefe, K. Kim, R. Merl, "Statistical Analysis of the Magnet Data for the Advanced Photon Source Storage Ring Magnets," in Particle Accelerator Conference, (Dallas, Texas, 1995). 